\title{
Spectral binning of cervicovaginal fluid metabolites improves prediction of spontaneous preterm birth and Lactobacillus species dominance
}

\author{
Emmanuel Amabebe1,*, Steven Reynolds2,* and Dilly O C Anumba1 \\ ${ }^{1}$ Academic Unit of Reproductive and Developmental Medicine, Department of Oncology and Metabolism, University of Sheffield, Sheffield, UK \\ ${ }^{2}$ Academic Unit of Radiology, Department of Infection, Immunity and Cardiovascular Disease, University of Sheffield, Sheffield, UK
}

Correspondence should be addressed to D O C Anumba: d.o.c.anumba@sheffield.ac.uk

*(E Amabebe and S Reynolds contributed equally to this work)

\section{Lay summary}

Health-promoting bacteria (lactobacilli) exist in harmony with the vaginal environment. They are the predominant vaginal bacterial species during pregnancy. However, the possibility of infection and inappropriate immune response are linked with unprompted preterm delivery (PTD). Other invasive lactobacilli can alter the chemical environment of the vagina as they seek to promote their growth. This study measured the change in concentration of biochemical compounds and predominant bacterial species in vaginal fluid that are linked to PTD. The study recruited 300 healthy pregnant women who provided vaginal fluid samples during the second trimester. The women who harboured more of Lactobacillus jensenii over Lactobacillus crispatus (both reported as health-promoting bacteria) in their vaginal fluid had less lactate and glutamate and experienced more PTD. This suggests that lactate and glutamate levels in vaginal fluid may have clinical application in identifying which Lactobacillus species is most active. These chemical biomarkers could provide quick and accurate prediction of PTD risk in clinical settings.

Key Words: - preterm delivery $\quad$ vaginal microbiota $\quad$ microbiota community state

type lactobacilli $\bullet{ }^{1} \mathrm{H}-\mathrm{NMR} \vee$ metabolomics

Reproduction and Fertility (2021) 2 L4-L6

The connection between vaginal microbiota-metabolite profiles and preterm delivery (PTD, birth <37 weeks' gestation) has been a topical subject. Previous research has focused on specific metabolites using proton NMR spectroscopy ( $\left.{ }^{1} \mathrm{H}-\mathrm{NMR}\right)$ (Amabebe et al. 2016, Stafford et al. 2017, Ansari et al. 2020). Spectral binning, that is, partitioning the spectrum into contiguous regions (bins), does not require prior knowledge of specific metabolites and is amenable to multivariate statistical analyses (Emwas et al. 2018).

This study retrospectively applied spectrual binning to ${ }^{1} \mathrm{H}-\mathrm{NMR}$ spectra of cervicovaginal fluid (CVF) taken from
300 consented asymptomatic high-risk pregnant women at $19+0$ to $22^{+6}$ weeks' gestation. After delivery (term $=250$ and preterm $=50$ ), we examined its potential to identify metabolic features associated with spontaneous PTD (sPTD) and microbiota community state types (CST).

CSTs were determined for 83 of these samples by sequencing the V1-V3 region of the 16S rRNA gene (Stafford et al. 2017). All ${ }^{1} \mathrm{H}-\mathrm{NMR}$ spectra were binned at $0.02 \mathrm{ppm}$, with differences between term/PTD or CST groups assessed by receiver operating characteristic (ROC) curve and partial least squares discriminant analysis (PLS-DA) (www. metaboanalyst.ca). 
Table 1 Summary of analysis for CST group (I, II, III, V) showing dominant Lactobacillus species and prevalence of preterm delivery in asymptomatic women studied at $19^{+0}-22^{+6}$ weeks' gestation. The median gestational age at birth in the overall cohort was 40 (37-43) weeks (term); and $33\left(21-36^{+6}\right)$ weeks (preterm). Comparisons for prevalence of sPTD were made between each CST group and the total cohort (i.e. including those where CST had not been determined, $n=300$ ), the subset of women with defined CSTS $(n=83)$, and women in CST-I $(n=26)$. No CST-IV women were identified in our study. Within individual CST groups, spectrum bin intensities were not compared for term vs. preterm women due to relatively small sample populations, e.g. only 2 and 3 women delivered preterm in the CST-I and CST-II groups respectively. Participants were recruited from the Jessop Maternity Wing of the Royal Hallamshire Hospital, Sheffield, UK. Their demographic details including age, ethnicity, previous PTD, smoking, vaginal $\mathrm{pH}$, and cervical length are as published previously (Stafford et al. 2017).

\begin{tabular}{|c|c|c|c|c|c|}
\hline CST & Dominant species & $n$ & Term & Preterm & Prevalence of PTD (\%) \\
\hline I & L. crispatus & 26 & 24 & 2 & 7.6 \\
\hline II & L. gasseri & 10 & 7 & 3 & 30.0 \\
\hline III & L. iners & 34 & 28 & 6 & 17.6 \\
\hline V & L. jensenii & 11 & 6 & 5 & $45.5^{*}$ \\
\hline III/V & L. iners/L. jensenii & 2 & 1 & 1 & NA \\
\hline Undetermined & NA & 217 & 184 & 33 & 15.2 \\
\hline Total, $n$ & NA & 300 & 250 & 50 & 16.7 \\
\hline
\end{tabular}

*Prevalence of SPTD significantly higher in the CST-V women than in CST-I women $(P=0.02)$, and total study cohort including women with undetermined $\operatorname{CSTs}(P=0.03)$.

CST, community state type; SPTD, spontaneous preterm delivery; $n$, sample population in the subset; Undetermined, no CSTs; NA, not applicable.

There were no significant differences in demographics between term and preterm women in the overall $(n=300)$ or CST-determined $(n=83)$ cohort (Stafford et al. 2017). sPTD rates in the CST-determined (20.5\%) and total cohort (16.7\%) were not significantly different $(P=0.42)$. However, women with CST-V (Lactobacillus jensenii-dominated) had higher sPTD rates compared to those with CST-I (Lactobacillus crispatusdominated) (Table 1).

From ${ }^{1} \mathrm{H}-\mathrm{NMR}$ spectral binning, lactate (bins 1.28 and $1.30 \mathrm{ppm}$ ) was higher in term than preterm women both for the overall cohort $(P<0.0001)$ and CST-determined women $(P<0.0001)$. Similarly, unassigned bins at 3.61, 3.67 and 3.69 ppm were higher in preterm than termdelivered women (all $P<0.0001$ ). Irrespective of pregnancy outcome, lactate had the highest variable importance in projection scores in differentiating the dominant CST of women. L. crispatus-dominated CST-I showed higher lactate $(P<0.0001)$ and glutamate $(P=0.007)$ bin integrals compared to other CST groups, whereas L. gasseri-dominated CST-II and L. jensenii-dominated CST-V had a higher bin integral at 3.83 ppm compared to other CST groups $(P=0.003)$. Summing lactate and glutamate bins improved the ROC discrimination for CST-I vs CST-V (Table 2).

Similar to previous observations, both lactate, glutamate and unidentified bins (3.61, 3.67 and 3.69 ppm) show potential as predictors of dysbiosis/infection (Ceccarani et al. 2019) and sPTD (Stafford et al. 2017), that is, high levels of lactate and glutamate indicate L. crispatus dominance over L. jensenii. Future studies using 2D NMR methods could identify other metabolites associated with these differences (3.61, 3.67 and 3.69 ppm).

CVF lactate and glutamate levels may have clinical application in providing a quick, accurate and reliable snapshot of the functional activity of the predominant vaginal Lactobacillus spp. However, the PLS-DA data

Table 2 Predictive capacity of lactate and glutamate $1 \mathrm{H}$-NMR spectrum bins for asymptomatic pregnant women with L. crispatus (CST-I) vs. L. jensenii-dominated (CST-V) vaginal microbiota.

\begin{tabular}{lll}
\hline Metabolite, bins (ppm) & AUC \\
\hline Lactate, 1.28 & & 0.84 \\
Lactate, 1.30 & 0.82 \\
Glutamate, 2.38 & 0.81 \\
Lactate, 4.11 & 0.84 \\
All & 0.94 \\
\hline
\end{tabular}

\begin{tabular}{c}
\hline Sensitivity (\%) \\
\hline 96.2 \\
84.6 \\
65.4 \\
76.9 \\
96.2 \\
\hline
\end{tabular}

\begin{tabular}{c}
\hline Specificity $(\%)$ \\
\hline 72.7 \\
81.8 \\
100 \\
90.9 \\
81.8 \\
\hline
\end{tabular}

\begin{tabular}{|c|c|}
\hline PPV (\%) & NPV $(\%)$ \\
\hline 89.3 & 88.9 \\
\hline 91.7 & 69.2 \\
\hline 100 & 55.0 \\
\hline 95.2 & 62.5 \\
\hline 92.6 & 90.0 \\
\hline
\end{tabular}

CST, community state type; ${ }^{1} \mathrm{H}-\mathrm{NMR}$, proton nuclear magnetic resonance spectroscopy; All, sum of lactate (1.28, 1.30 and $\left.4.11 \mathrm{ppm}\right)+\mathrm{glutamate}(2.38 \mathrm{ppm})$ bins; AUC, area under the ROC curve; PPV, positive predictive value; NPV, negative predictive value; ppm, parts per million

https://raf.bioscientifica.com

https://doi.org/10.1530/RAF-21-0065 (c) 2021 The authors Published by Bioscientifica Ltd

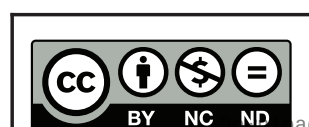

This work is licensed under a Creative Commons Attribution-NonCommercial-NoDerivatives 4.0 International License.
ded from Bioscientifica.com at $04 / 26 / 2023$ 01:28:48PM 
showed a high degree of overlap for CST-II, -III and -V groups making it difficult to distinguish these groups by metabolite profile alone - only CST-I appeared to be separated.

Although the vaginal microbiota may increasingly be dominated by lactobacilli during pregnancy, the risk of infection-inflammation-associated spontaneous PTD may still be substantial depending on the predominant species.

\section{Declaration of interest}

The authors declare that there is no conflict of interest that could be perceived as prejudicing the impartiality of the research reported.

\section{Funding}

This study was funded by the Medical Research Council UK Development Pathways Funding Scheme (DPFS) (Grant number: MR/J014788/1).

\section{Ethics approval}

This study was reviewed and approved by the Yorkshire \& Humber (Sheffield) Committee of the UK National Research Ethics Service (REC Number 13/YH/0167).

\section{Author contribution statement}

E A and S R designed the study. E A, S R and D A all contributed to writing the manuscript. S R and E A processed and analysed the ${ }^{1} \mathrm{H}-\mathrm{NMR}$ metabolite data. D A collected some of the cervicovaginal fluid samples and collated patients' clinical data. All authors read and approved the final manuscript for submission.

\section{Acknowledgements}

The authors would like to thank all the women who consented to be part of these studies. The authors also thank Victoria Stern who assisted in collecting the cervicovaginal fluid samples and collating patients' clinical data, Graham Stafford, Jenifer Parker and James Kistler who assisted in processing the initial microbiota data, and Martyn Paley for his contribution to the ${ }^{1} \mathrm{H}-\mathrm{NMR}$ experiments.

\section{References}

Amabebe E, Reynolds S, Stern VL, Parker JL, Stafford GP, Paley MN \& Anumba DO 2016 Identifying metabolite markers for preterm birth in cervicovaginal fluid by magnetic resonance spectroscopy. Metabolomics 12 67. (https://doi.org/10.1007/s11306-016-0985-x)

Ansari A, Lee H, You YA, Jung Y, Park S, Kim SM, Hwang GS \& Kim YJ 2020 Identification of potential biomarkers in the cervicovaginal fluid by metabolic profiling for preterm birth. Metabolites 10 349. (https://doi.org/10.3390/metabo10090349)

Ceccarani C, Foschi C, Parolin C, D'Antuono A, Gaspari V, Consolandi C, Laghi L, Camboni T, Vitali B, Severgnini M, et al. 2019 Diversity of vaginal microbiome and metabolome during genital infections. Scientific Reports 9 14095. (https://doi.org/10.1038/ s41598-019-50410-x)

Emwas A-H, Saccenti E, Gao X, McKay RT, Dos Santos V, Roy R \& Wishart DS 2018 Recommended strategies for spectral processing and post-processing of 1D (1)H-NMR data of biofluids with a particular focus on urine. Metabolomics 14 31. (https://doi.org/10.1007/ s11306-018-1321-4)

Stafford GP, Parker JL, Amabebe E, Kistler J, Reynolds S, Stern V, Paley M \& Anumba DOC 2017 Spontaneous preterm birth is associated with differential expression of vaginal metabolites by lactobacilli-dominated microflora. Frontiers in Physiology 8615. (https://doi.org/10.3389/fphys.2017.00615)

Received in final form 2 November 2021

Accepted 4 November 2021

Accepted Manuscript published online 4 November 2021 (c) 2021 The authors Published by Bioscientifica Ltd
This work is licensed under a Creative Commons Attribution-NonCommercial-NoDerivatives 4.0 International License. ded from Bioscientifica.com at $04 / 26 / 2023$ 01:28:48PM 